\title{
Localized relaxation in a glass and the minimum in its orientational polarization contribution
}

\author{
G. P. Johari \\ Department of Materials Science and Engineering, McMaster University, Hamilton, \\ Ontario, Canada, L8S 4 L7
}

\author{
G. Power and J. K. Vij ${ }^{\mathrm{a})}$ \\ Laboratory of Advanced Materials, Department of Electronic and Electrical Engineering, Trinity College, \\ University of Dublin, Dublin 2, Ireland
}

(Received 6 March 2002; accepted 23 April 2002)

\begin{abstract}
The dielectric permittivity and loss spectra of the glassy state of 5-methyl-2-hexanol obtained by quenching it from the liquid state has been studied. In one experiment, the spectra were studied at different temperatures as the quenched sample was heated at $0.1 \mathrm{~K} / \mathrm{min}$ from 105.3 to $160.5 \mathrm{~K}$. In the second experiment, the quenched sample was heated from 77 to $131.6 \mathrm{~K}$ and kept at that temperature for $14.6 \mathrm{ks}$. The relaxation rate, $f_{m, \beta}$, the dielectric relaxation strength, $\Delta \epsilon_{\beta}$, and the distribution of relaxation time parameters, $\alpha$ and $\beta$, for the Johari-Goldstein process were determined. The parameter $\beta$ was found to be equal to 1 and independent of both the temperature and time, $\Delta \epsilon_{\beta}$ initially decreased on increasing the temperature, reached a minimum value at $\sim 145.6 \mathrm{~K}$, and then increased. The plot of $f_{m, \beta}$ against the reciprocal temperature decreased in slope and at $\sim 140 \mathrm{~K}$ became linear. This indicates that $f_{m, \beta}$ increases on structural relaxation. In the course of the annealing at $131.6 \mathrm{~K}, \Delta \epsilon_{\beta}$ of the quenched sample decreased with time, approaching a plateau value. It is described by an equation, $\Delta \epsilon_{\beta}(t)=\Delta \epsilon_{\beta}(t \rightarrow \infty)+\left[\Delta \epsilon_{\beta}(t=0)-\Delta \epsilon_{\beta}(t\right.$ $\rightarrow \infty)] \exp [-(t / \tau)]$, where $t$ is the time, and $\tau(=3.5 \mathrm{ks})$ is the characteristic time. It is pointed out that contrary to the earlier finding, $o$-terphenyl shows a $\beta$ relaxation in the equilibrium liquid state. A consideration of dielectric permittivity arising from small-angle motions of all molecules, which has been suggested as an alternative mechanism for the localized motions seen as $\beta$ relaxation, indicates that this mechanism is inconsistent with the known increase in the equilibrium permittivity on cooling. (C) 2002 American Institute of Physics. [DOI: 10.1063/1.1485960]
\end{abstract}

\section{INTRODUCTION}

There are two characteristics of the glassy state, (i) its physical properties change with time at a rate that increases with the temperature of the glass, and (ii) localized molecular motions occur in its disordered structure. The first is a result of structural relaxation ${ }^{1,2}$ during which a glass ${ }^{3}$ spontaneously approaches the state of its equilibrium liquid, and its volume, enthalpy, and entropy decrease with time asymptotically, and its refractive index, vibrational frequencies, viscosity, and relaxation time increase. The second characteristic refers to the secondary, or $\beta$ relaxation in the otherwise rigid state of a glass. Also called the Johari-Goldstein relaxation, these motions are also found to be a property of the equilibrium liquid state, and are observed in dielectric, mechanical, and light scattering spectroscopy as well as by NMR and other measurements in which molecular diffusion may be detected. A review by Rössler and co-workers, ${ }^{4}$ who have contributed significantly to the understanding of the localized motions by dielectric and NMR experiments may be consulted for details.

In a low-viscosity liquid, only one relatively narrow dielectric relaxation peak has been observed. ${ }^{5}$ As a liquid is cooled and its viscosity and density increase, a new mode of

${ }^{\text {a)} E l e c t r o n i c ~ m a i l: ~ j v i j @ t c d . i e ~}$ molecular motion, called the $\alpha$-relaxation process emerges. Its rate rapidly decreases on supercooling and ultimately, at a certain temperature, becomes comparable to one's measurement time scale. At this temperature, molecular diffusion associated with the $\alpha$-relaxation process becomes kinetically frozen, and the liquid is said to have vitrified. The $\beta$-relaxation process persists in the vitrified state. ${ }^{6}$ Thus, molecular diffusion in a low-viscosity liquid occurs mainly by the mechanism of the $\beta$-relaxation process, and at high viscosity it occurs by the mechanisms of both the $\beta$-, and the $\alpha$-relaxation processes. ${ }^{7,8}$ In glasses, $\beta$ relaxation alone is observed in the usual laboratory time scale experiments. Thus, the contribution to permittivity due to orientational polarization in the low-viscosity liquid is only due to the $\beta$-relaxation process, and in a viscous liquid and glass, it is equal to the sum of the contributions to permittivity from the $\alpha$, and $\beta$-relaxation processes, or $\left(\Delta \epsilon_{\alpha}+\Delta \epsilon_{\beta}\right)$, with dielectric relaxation strengths of $\Delta \epsilon_{\alpha}$ and $\Delta \epsilon_{\beta}$, respectively. ${ }^{5,7} \mathrm{In}$ certain theories, localized molecular motions of the $\beta$ process are seen to be the precursor of the $\alpha$ relaxation. ${ }^{9,10}$ These motions also contribute to the entropy of the glass. ${ }^{11-13}$ These have been connected ${ }^{14}$ to the non-Debye temperature dependence of the heat capacity at $T<1 \mathrm{~K},{ }^{15}$ and the onset of their molecular motions on heating has been found to occur over a broad temperature range, with a 
sigmoid-shape heat capacity increase resembling that observed on glass softening. ${ }^{16-18}$ The effect of electrolytes on the magnitude of their dielectric relaxation has been studied. ${ }^{19}$ These localized motions have been found to cause nucleation and crystallization of some glasses at $T<T_{g} .{ }^{20,21}$

When a liquid is vitrified by rapid cooling, its glassy state is bulkier and its $\Delta \epsilon_{\beta}$ is expected to be high, and if it is vitrified by slow cooling, its glassy state is denser, and its $\Delta \epsilon_{\beta}$ is expected to be low. It is also expected that densification would alter the relaxation rate of the $\beta$ process. Evidence for this has been found for some glasses in some experiments, ${ }^{22,23}$ but not in all glasses and other experiments. ${ }^{5,6,23-27}$ Structural relaxation of a glass has been found to decrease its $\Delta \epsilon_{\beta}$, as the state of its equilibrium liquid is approached at a constant temperature in all cases. ${ }^{6,22-27}$ Thus, although the extent of thermodynamic metastability, as measured by the frozen-in volume, enthalpy, and entropy of a glass, raises $\Delta \epsilon_{\beta}$, the $\beta$ process itself remains a property of the liquid state.

Since the total contribution to permittivity due to orientation polarization, $\Delta \epsilon\left(=\Delta \epsilon_{\alpha}+\Delta \epsilon_{\beta}\right)$, increases with an increase in density ${ }^{28-31}$ and $\Delta \epsilon_{\beta}$ decreases, structural relaxation should increase $\Delta \epsilon_{\alpha}\left(=\Delta \epsilon-\Delta \epsilon_{\beta}\right)$, by an amount that would be greater than that expected from the density increase alone. This amount is equal to the decrease in $\Delta \epsilon_{\beta}$ on structural relaxation. Thus the $\alpha$-relaxation process is expected to grow at the expense of the $\beta$-relaxation process as the density of the glass increases at a fixed temperature.

Here we report a study of changes in the $\beta$-relaxation process on spontaneous structural relaxation of the glassy state of an uncrystallizable liquid, 5-methyl-2-hexanol under two conditions, (i) on heating at a fixed rate, and (ii) isothermally. We then examine the characteristics of the $\beta$-relaxation process in the equilibrium liquid and glassy states, and discuss the results in terms of the combined time and temperature effects, and briefly describe the implication of these observations for the potential energy landscape of the $\beta$-relaxation process. Dielectric relaxation features of 5-methyl-2-hexanol as a function of temperature have been reported before. ${ }^{32}$

\section{EXPERIMENTAL METHODS}

5-methyl-2-hexanol was of purum grade, purchased from Fluka AG., Switzerland, and used as such. The dielectric cell was a miniature parallel plate condenser with 18 plates and a nominal capacitance of $27 \mathrm{pF}$. This capacitance was accurately measured in air prior to the use of the capacitor. The sample was contained in a vial of $10 \mathrm{~mm}$ diam 33 $\mathrm{mm}$ length, in which a $100 \Omega$ platinum resistance temperature sensor and the condenser were immersed. The assembly was mounted inside a cryostat, purchased from Oxford Instruments. The cryostat was thermostatted using a temperature controller (model ITC502, also by Oxford). The temperature was programmed for heating at a rate of $0.1 \mathrm{~K} / \mathrm{min}$, and the $\epsilon^{\prime}$ and $\epsilon^{\prime \prime}$ spectra were obtained in the frequency range of $10 \mathrm{~Hz}-1 \mathrm{MHz}$ by means of a Solartron FRA-1255A frequency response analyzer interfaced with a Chelsea dielectric interface. The spectra were taken in a period of $\sim 218 \mathrm{~s}$, during which the temperature would have increased

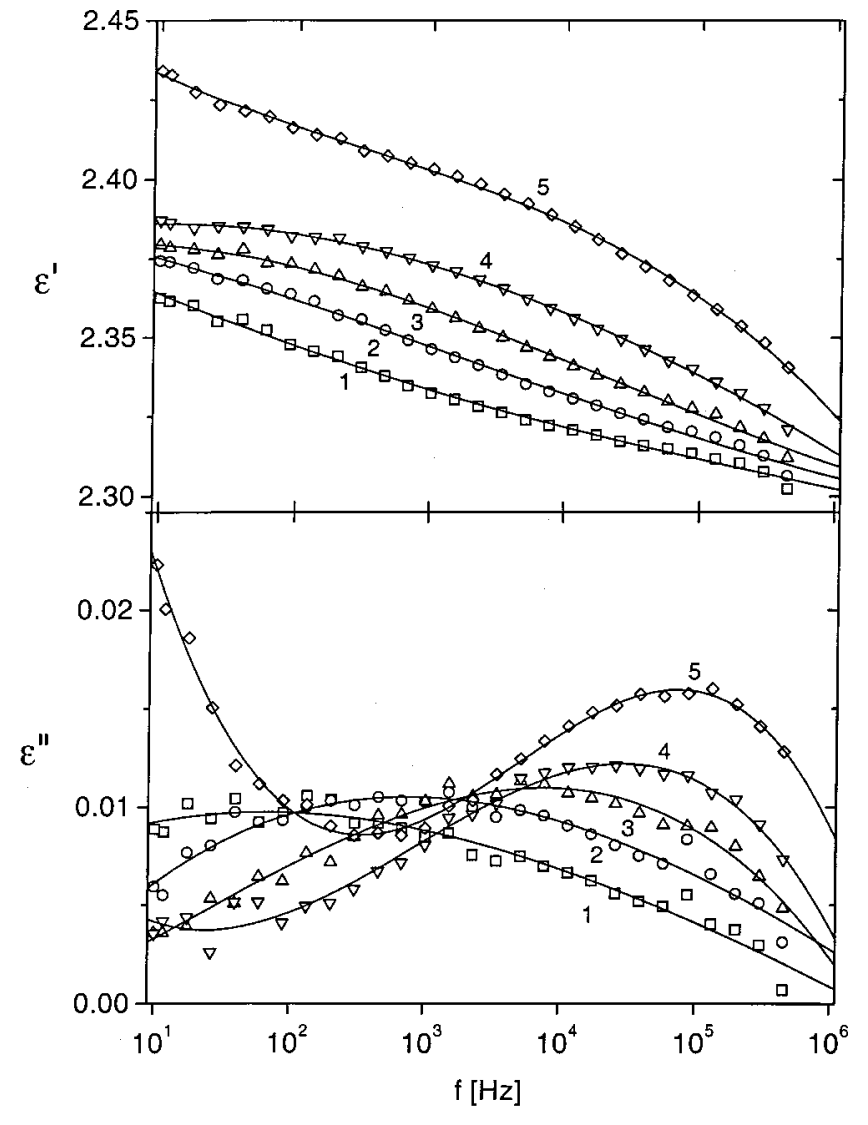

FIG. 1. The $\epsilon^{\prime}$ and $\epsilon^{\prime \prime}$ spectra of 5-methyl-2-hexanol at several temperatures below and above its $T_{g}$. Curves $1-5$ are for $116.7,125.5,133.1,142.3$, and $151.7 \mathrm{~K}$.

by $\sim 0.36 \mathrm{~K}$ due to the heating rate already set for this experiment. The temperature reported here is the mean of $\sim 5$ temperatures measured during each spectral scan. Two sets of experiments were performed. In one set, the sample was quenched by immersing in liquid nitrogen, transferred to the temperature control assembly and its $\epsilon^{\prime}$ and $\epsilon^{\prime \prime}$ spectra were measured continuously during heating at $0.1 \mathrm{~K} / \mathrm{min}$. In the second set, the sample was quenched by immersing in liquid nitrogen and then transferred to the temperature control unit. There it was heated rapidly to $131.6 \mathrm{~K}$, the temperature stabilized for $\sim 30 \mathrm{~min}$, and its $\epsilon^{\prime}$ and $\epsilon^{\prime \prime}$ spectra measured continuously while keeping the sample at $131.6 \mathrm{~K}$ over a period of $14.6 \mathrm{ks}$.

\section{RESULTS}

Typical spectra of $\epsilon^{\prime}$ and $\epsilon^{\prime \prime}$ obtained in the first set of the study are shown for five temperatures in Fig. 1. Their shapes show a broad steplike decrease in $\epsilon^{\prime}$ and a broad peak in $\epsilon^{\prime \prime}$. The shapes of the relaxation spectra have been analyzed in different manners by different groups. However, for an unbiased analysis of the data, we use the Havriliak- and Negamitype equations, ${ }^{27}$ which fits the data to several relaxation processes by minimizing the deviation of the data from the fits. It was done in the manner described earlier, ${ }^{32}$ by fitting the following equation: ${ }^{33}$ 


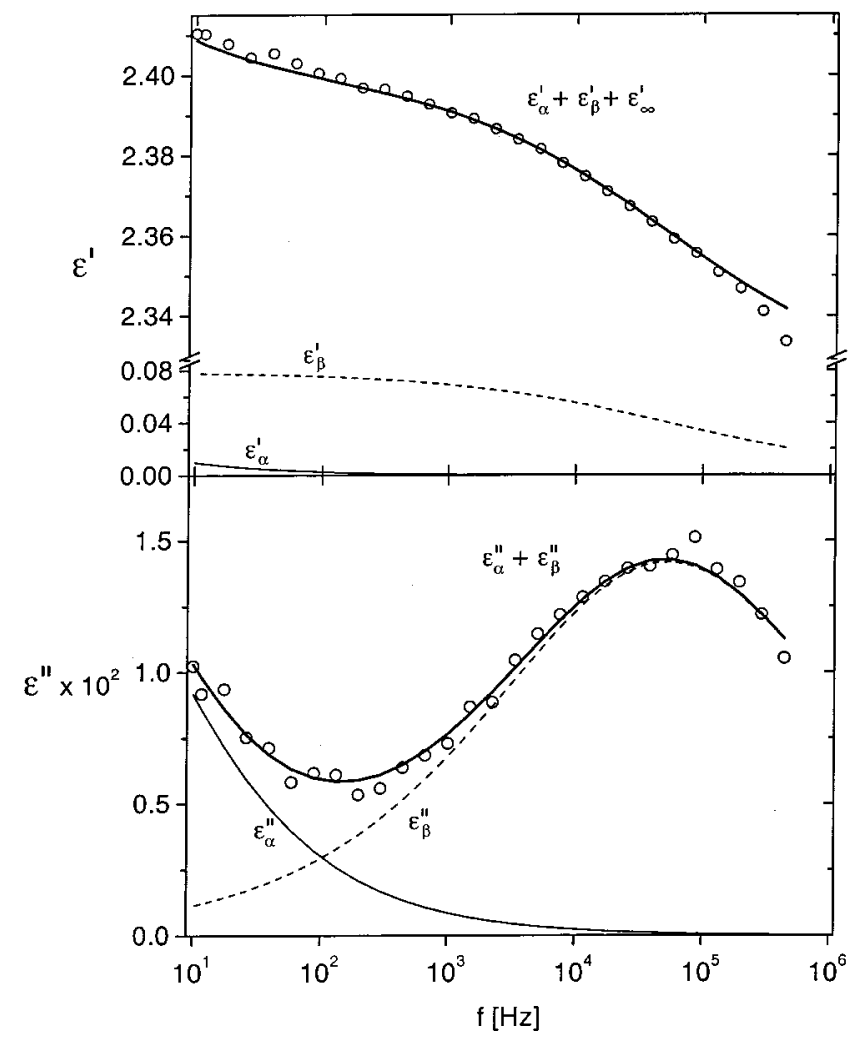

FIG. 2. The resolution of a typical spectra measured at $149.5 \mathrm{~K}$ for obtaining $\Delta \boldsymbol{\epsilon}_{\beta}$ and $f_{m, \beta}$ and the $\alpha$ and $\beta$ parameters.

$$
\epsilon^{*}(\omega)=\epsilon_{\infty}+\frac{\Delta \epsilon_{\alpha}}{\left[1+\left(j \omega \tau_{\alpha}\right)^{\alpha_{1}}\right]^{\beta_{1}}}+\frac{\Delta \epsilon_{\beta}}{\left[1+\left(j \omega \tau_{\beta}\right)^{\alpha_{2}}\right]^{\beta_{2}}},
$$

to the data. Here $\Delta \epsilon_{\alpha}$ is the dielectric relaxation strength of the $\alpha$ process, $\tau_{\alpha}$ is its relaxation time, $\omega$ is the angular frequency $[=2 \pi f(f$ is the frequency in $\mathrm{Hz})]$, and $\alpha_{1}$ and $\beta_{1}$ are the two parameters of the Cole-Cole and Cole-Davidson distribution functions. Similarly, $\Delta \epsilon_{\beta}$ is the dielectric relaxation strength of the $\beta$ process, $\tau_{\beta}$ is its relaxation time, and $\alpha_{2}$ and $\beta_{2}$ are the corresponding two parameters. From fitting the equation to the $\epsilon^{\prime}$ and $\epsilon^{\prime \prime}$ spectra, only $\Delta \epsilon_{\beta}, \tau_{\beta} \alpha_{2}$, and $\beta_{2}$ were reliably obtained. A detailed analysis of the dc conductivity of 5-methyl-2-hexanol, and the characteristics of its $\alpha$ - and $\beta$-relaxation processes had previously been carried out in a separate study, which may be consulted for details. $^{32}$

A typical fit of Eq. (1) to the $\epsilon^{\prime}$ and $\epsilon^{\prime \prime}$ spectra of the $\beta$-relaxation process is shown in Fig. 2, where the full lines indicate the resolved $\epsilon^{\prime}$ dispersion and $\epsilon^{\prime \prime}$ peak. As has been discussed earlier, ${ }^{26}$ the spectra obtained over this temperature range correspond to process III, and hence the quantities obtained correspond to $\Delta \epsilon_{\mathrm{III}}$, and $f_{m, \mathrm{III}}$ (frequency of maximum $\epsilon^{\prime \prime}$, which is used as a general measure of relaxation rate) in the earlier study. This relaxation process has been identified already as the Johari-Goldstein relaxation process. For brevity here, the subscript "III" is dropped and the terms are written as $\Delta \epsilon_{\beta}$ and $f_{m, \beta}$ for this relaxation process, and the parameters $\alpha_{3}$ and $\beta_{3}$ are written as $\alpha$ and $\beta$. Its relaxation rate, $f_{m, \beta}$, was obtained from the equation

$$
f_{m, \beta}=\left(2 \pi \tau_{\beta}\right)^{-1}\left[\sin \left(\frac{\alpha \pi}{2+2 \beta}\right)\right]^{1 / \alpha}\left[\sin \left(\frac{\alpha \beta \pi}{2+2 \beta}\right)\right]^{-1 / \alpha} .
$$

The highest-temperature spectra of Fig. 1 (curve 5) is for a temperature $(151.7 \mathrm{~K})$ above the $T_{g}$, while curve $2(125.5$ $\mathrm{K})$ is below the $T_{g}$, which is $\sim 148 \mathrm{~K}$ for 5-methyl-2hexanol. Peaks in the $\epsilon^{\prime \prime}$ spectra at frequencies higher than the frequency of the $\alpha$-relaxation peak in this temperature range have been observed before ${ }^{32}$ and shown to be due to the Johari-Goldstein relaxation. The increasing $\epsilon^{\prime \prime}$ with decreasing frequency below $500 \mathrm{~Hz}$ for curve 5 in Fig. 1, and below $100 \mathrm{~Hz}$ for the $\epsilon^{\prime \prime}$ spectra in Fig. 2, is the highfrequency tail of the $\alpha$-relaxation process. In some cases, where this tail has been found to contain contributions to $\epsilon^{\prime \prime}$ other than those from the $\alpha$-relaxation process, and a peak at higher frequencies has not been clearly observed, this tail has been called "the wing." In that connection it should be noted that this tail or "wing" (appearing in the low-frequency region of the $\epsilon^{\prime \prime}$ spectra in Figs. 1 and 2) cannot be due to the dc conductivity for two reasons: (i) the slope of the plot of $\log \epsilon^{\prime \prime}$ against $\log f$ in the region of this "wing" is not unity; and (ii) this "wing" turns into the high-frequency part of the $\alpha$-relaxation peak at higher temperatures, which has already been shown in Ref. 32, and therefore is not shown in Figs. 1 and 2 here. After the $\alpha$-relaxation peak has appeared, and at still lower frequencies, contributions from the dc conductivity are expected to raise $\epsilon^{\prime \prime}$. This $\alpha$-relaxation process has been described earlier in detail, ${ }^{32}$ where the "wing" was not found. Thus, there is little doubt that the $\epsilon^{\prime \prime}$ peak observed in Fig. 1 is due to the Johari-Goldstein relaxation. It is superimposed on the high-frequency tail of the $\alpha$-relaxation process, and its contribution approaches zero, as seen in the lower panel of Fig. 2.

The distribution of relaxation time parameter, $\beta$, in our analysis was found to be equal to 1.00 at all temperatures, but the value of the parameter $\alpha$ varied. Its value is plotted against the temperature, $T$, in Fig. 3(A). It increases from 0.25 at $115.3 \mathrm{~K}$ to 0.48 at $159.1 \mathrm{~K}$. The value of $\Delta \epsilon_{\beta}$ is plotted against $T$ in Fig. 3(B). It decreases from 0.105 at $115.3 \mathrm{~K}$ to 0.073 at $145.6 \mathrm{~K}$, and thereafter increases to 0.106 at $159.1 \mathrm{~K}$, thus showing a minimum. The value of $f_{m, \beta}$ is plotted against the reciprocal temperature in Fig. 4. It shows a slight curvature and a change in the slope at $\sim 140$ K.

For studying the time dependence of $\alpha, \beta, \Delta \epsilon_{\beta}$, and $f_{m, \beta}$ in the second set of experiments, the 5-methyl-2hexanol sample was kept at $131.6 \pm 0.04 \mathrm{~K}$, and its spectra measured continuously up to a total elapsed time of $14.6 \mathrm{ks}$. The parameter $\beta$ for the spectra, as obtained from the analysis was found to remain constant at 1.00. The values of $\alpha$, $\Delta \epsilon_{\beta}$, and $f_{m, \beta}$ thus obtained are plotted against the time, $t$, in Figs. 5(A), 5(B), and 5(C), respectively. Here $\alpha$ remains constant at $0.36 \pm 0.01 . \Delta \epsilon_{\beta}$ decreases from 0.082 to 0.074 according to the equation $\Delta \epsilon_{\beta}=0.0736+0.00825$ $\times[\exp (-t / 3463)]$, where $t$ is the time in $\mathrm{s}$. The relaxation rate, $f_{m, \beta}$, shows little change, and remains at $3.9 \pm 0.3 \mathrm{kHz}$. The scatter in the data plotted in Fig. 5 was found to be random, mostly due to the errors in the analysis. The $f_{m, \beta}$ 


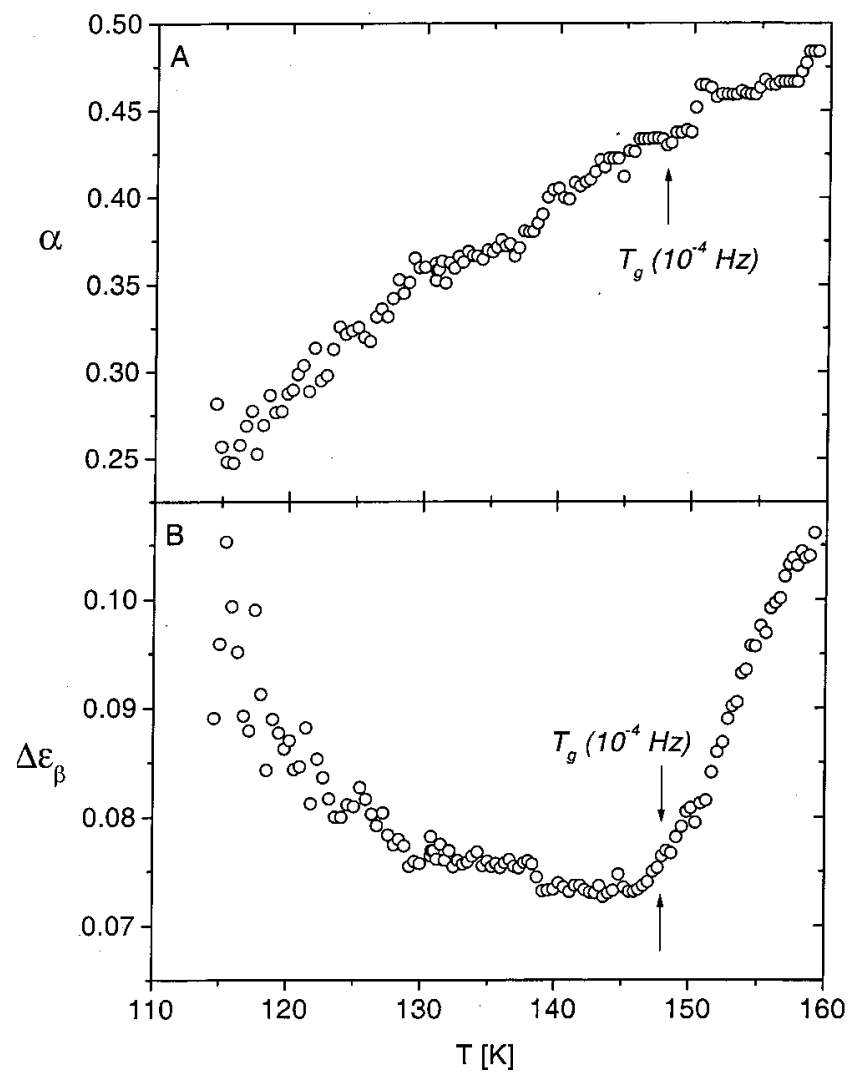

FIG. 3. (A) The value of $\alpha$ for the fastest relaxation process in 5-methyl-2hexanol is plotted against the temperature. (B) The dielectric relaxation strength $\Delta \epsilon_{\beta}$ of the fastest process in 5-methyl-2-hexanol is plotted against the temperature. Also marked on the plots is $T_{g}$ of $148.1 \mathrm{~K}$. It was obtained by extrapolating the dielectric relaxation rate of the $\alpha$ process to $10^{-4} \mathrm{~Hz}$ from the data in Ref. 32.

values, which are more sensitive to change in $T$ than $\Delta \epsilon_{\beta}$ and $\alpha$ however, could be correlated with the fluctuations in the temperature.

\section{DISCUSSION}

\section{A. Magnitude of orientation polarization due to localized motions}

It is well known that the magnitude of orientation polarization, $\Delta \epsilon$, of a material varies inversely with $T$ and directly with the number density of dipoles, $N_{d}$. As the density of a glass increases on cooling, the orientation polarization due to the $\beta$ process, $\Delta \epsilon_{\beta}$, of a glass is expected to increase approximately by an amount proportional to $N_{d} / T$. The ${ }^{2} \mathrm{H}$ NMR studies of several glasses appear to show that all molecules reorient by a small, temperature-independent angle. ${ }^{34,35}$ Accordingly, $\Delta \epsilon_{\beta}$ of a glass should increase with decrease in $T$. The finding here is the opposite, i.e., $\Delta \epsilon_{\beta}$ decreases on cooling a glass, in agreement with the earlier studies., ${ }^{5,622-27}$ As mentioned earlier here, the density of a glass increases on isothermal structural relaxation. Therefore, if the angle of reorientation of molecules did not change, as concluded recently ${ }^{34,35} \Delta \epsilon_{\beta}$ would increase on an increase in density during structural relaxation. Physical aging studies of glasses here and elsewhere, however, show the opposite, i.e., $\Delta \epsilon_{\beta}$ decreases on physical aging or annealing. ${ }^{6,7,22-27,36}$

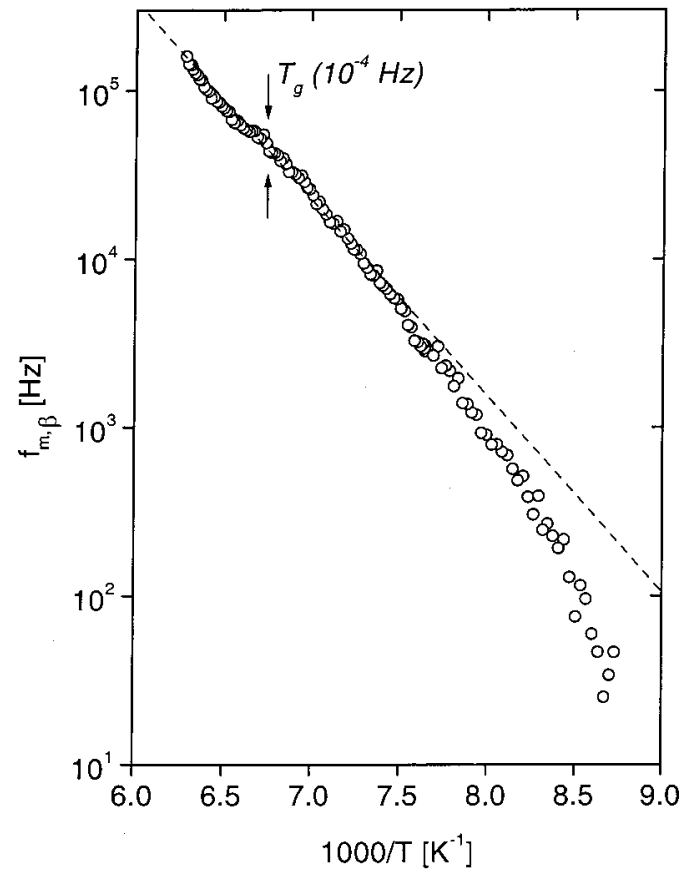

FIG. 4. The frequency of maximum dielectric loss in the spectra of the $\beta$-, or Johari-Goldstein relaxation process in 5-methyl-2-hexanol is plotted against the reciprocal temperature. The dashed line and its extension in the region, where the equilibrium state was not attained is a linear fit to the high-temperature data. $T_{g}$ (from extrapolation of dielectric data to $\left.10^{-4} \mathrm{~Hz}\right)=148.1 \mathrm{~K}$ (after Ref. 32) is marked on the plot.

\section{B. Effects of structural relaxation on localized motions}

In order to discuss the occurrence of the minimum in $\Delta \epsilon_{\beta}$ on heating at a fixed rate, as seen in Fig. 3(B), it seems necessary to consider first the characteristics of the $\beta$-relaxation process in relation to both the thermodynamic nonequilibrium state of a glass and the equilibrium state of its liquid. As mentioned in Sec. I, the volume, entropy, and enthalpy of a glass decrease and the refractive index, viscosity, and relaxation time increase on its structural relaxation. The rate of the $\alpha$-relaxation process decreases and the entire relaxation spectra shifts to lower frequencies. In experiments designed to determine the existence of localized motions in rigid molecular glasses, it was discovered that structural relaxation or slow cooling led to a decrease in the height of the $\beta$-relaxation peak observed in the dielectric loss. A small change in the $\beta$-relaxation peak temperature measured for a fixed frequency was also detected on structural relaxation of several glasses, but it was not certain whether that indicated a change in the $\beta$-relaxation rate or whether it resulted as an artifact of (i) a shift of the $\alpha$-relaxation process toward lower frequencies, and (ii) a decrease in the background dielectric loss. These effects were later resolved by measuring the dielectric spectra of the differently quenched glasses and physically aging a quenched glass. ${ }^{27}$ The results demonstrated that structural relaxation of a glass decreases $\Delta \epsilon_{\beta}$. Thus, like the volume, enthalpy, and entropy of a liquid, $\Delta \epsilon_{\beta}$ of a glass is also time dependent. It was also found that $\Delta \epsilon_{\beta}$ of an equilibrium liquid decreased on cooling, as it did of its nonequilibrium glassy state. Hence, the $\beta$-relaxation process was found to have two further characteristics: (i) $\Delta \epsilon_{\beta}$ de- 


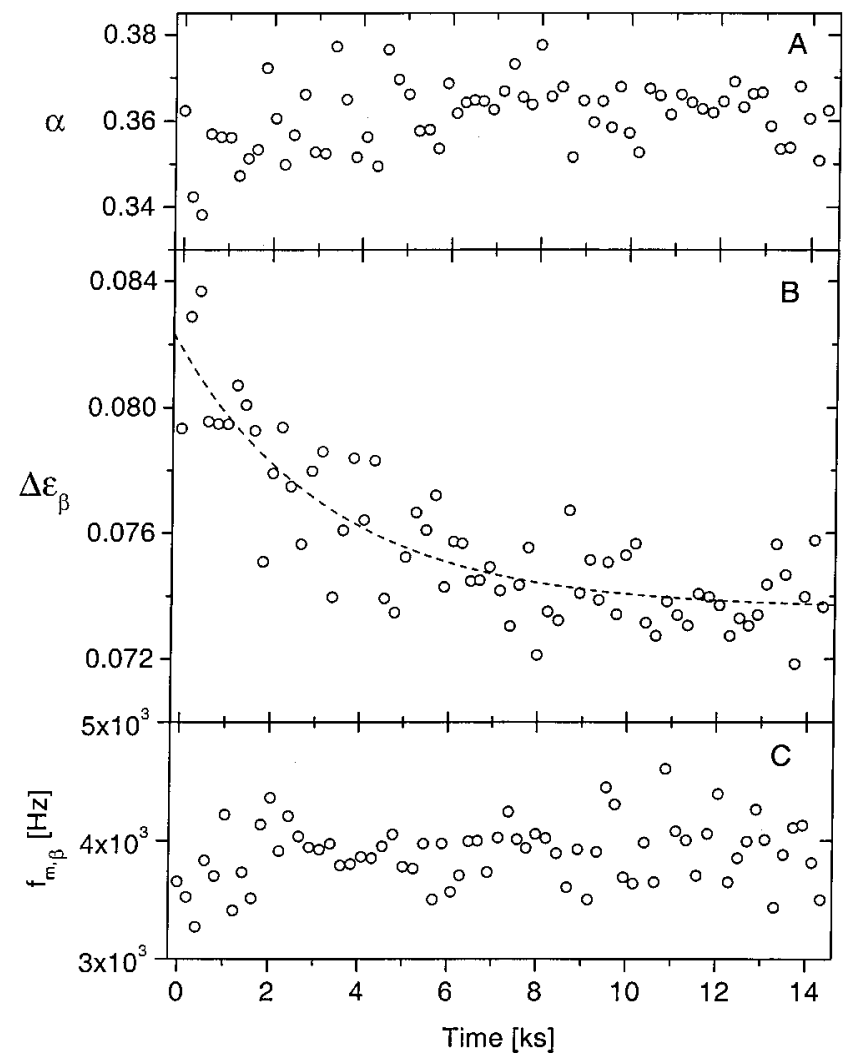

FIG. 5. (A) The value of $\alpha$ for the fastest relaxation process in 5-methyl-2hexanol is plotted against the time during which the quenched sample was kept at $131.6 \pm 0.04 \mathrm{~K}$. (B) The corresponding plot of the dielectric relaxation strength, $\Delta \epsilon_{\beta}$. The dashed line is the fit to the equation, $\Delta \epsilon_{\beta}(t)$ $=0.0736+0.00825 \exp [-(t / 3463)]$. (C) The corresponding plot of the frequency of maximum dielectric loss, $f_{m, \beta}$.

creases spontaneously on structural relaxation toward the equilibrium liquid value, and (ii) $\Delta \epsilon_{\beta}$ decreases as an equilibrium liquid is cooled, and $\Delta \epsilon_{\alpha}$ increases.

One more observation that bears on the nature of the $\beta$-relaxation process is worth discussing here. On the basis of a recent study, it has been suggested that the $\beta$-relaxation process in some cases may occur only in the quenched state of the glass, and that it may vanish on annealing the glass and it is not observed in the equilibrium liquid state. ${ }^{24,25}$ The suggestion had been based on the finding that the $\beta$-relaxation peak of magnitude $\epsilon^{\prime \prime} \sim 13 \times 10^{-5}$ observed in the quenched glassy state of $o$-terphenyl at $T \sim 205 \mathrm{~K}$ vanished after the $o$-terphenyl glass was annealed for $24 \mathrm{~h}$ at 234 $\mathrm{K}, 12 \mathrm{~K}$ below its glass-softening temperature, $T_{g}$, of $246 \mathrm{~K}$ (see the inset in Fig. 5 in Ref. 25). Since $o$-terphenyl apparently approached the equilibrium state on structural relaxation, it was concluded that there is no $\beta$-relaxation process in liquid $o$-terphenyl at $T>T_{g}$. Hence, it had been concluded that $o$-terphenyl is an exception as regards the $\beta$ relaxation in the liquid state, i.e., only its quenched glassy state shows the $\beta$ relaxation. ${ }^{25}$ After comparing the $\beta$-relaxation characteristics of $o$-terphenyl against those of D-sorbitol, it was further proposed that there may be two types of glasses, one in which $\beta$ relaxation was observed both in the equilibrium liquid and the glassy states, which was called the L-type relaxation, and a second in which it was observed only in the quenched glassy state but not in the equilibrium liquid state, which was called the G-type relaxation. ${ }^{25}$

The above-given conclusion conflicts with the accepted view that $\beta$ relaxation is an intrinsic property of the liquid state, and that it is a precursor of the $\alpha$-relaxation process, which emerges after the density and viscosity of a liquid has reached a certain high value on supercooling. For these reasons, we reconsider the earlier data, ${ }^{25}$ as follows: In Fig. 3 of that study, ${ }^{25}$ the plots for $T=250 \mathrm{~K}$ and $246 \mathrm{~K}$ show a highfrequency shoulder that curves downward and that seems to separate out as a $\beta$-relaxation peak at $T<246 \mathrm{~K}$. (Note that the curvature of this shoulder indicates that it is not the same as the high-frequency wing.) Since the data acquisition time for the spectra at a single temperature in that study was around $1 \mathrm{~h}(3.6 \mathrm{ks}),{ }^{25}$ and the dielectric relaxation time at $246 \mathrm{~K}$ is $0.1 \mathrm{ks}, o$-terphenyl at 250 and at $246 \mathrm{~K}$ would have been an equilibrium liquid. Also, the shapes of the spectra in the high-frequency region in the equilibrium liquid at 250 and $246 \mathrm{~K}$ (Fig. 3, Ref. 25) are qualitatively similar to the shapes of the spectra of D-sorbitol at 284 and $280 \mathrm{~K}$ (Fig. 6 in Ref. 25). All these observations show that, contrary to the earlier conclusions, $\beta$ relaxation does occur in the equilibrium liquid state of $o$-terphenyl. It is also worth pointing out that in the study of $o$-terphenyl, ${ }^{24}$ particularly in the $\epsilon^{\prime}$ and $\epsilon^{\prime \prime}$ plots in Fig. 6 of Ref. 24, $\epsilon^{\prime \prime}$ showed a peak at about $200 \mathrm{~K}$, but $\epsilon^{\prime}$ continued to increase with decreasing temperature. This is quite the opposite of the known dielectric relaxation behavior of materials, namely, that dielectric relaxation decreases $\epsilon^{\prime}$ and that $\left(d \epsilon^{\prime} / d T\right)$ is negative at a temperature where $\epsilon^{\prime \prime}$ shows a peak. ${ }^{28-31}$ Moreover, this behavior conflicts with the Kramers-Kronig relation applied to the complex permittivity at each temperature.

It is also noteworthy that the apparent vanishing of the $\beta$-relaxation peak on annealing a quenched sample of $o$-terphenyl at $234 \mathrm{~K}^{25}$ is contrary to the recent finding that the $\beta$-relaxation peak that could not be found in the $\epsilon^{\prime \prime}$ spectra (or fixed frequency measurements of the quenched samples), became observable after a glass was structurally relaxed by annealing at $T<T_{g} .{ }^{37}$ This decreased the $\epsilon^{\prime \prime}$ contribution from the $\alpha$-relaxation process at those frequencies where the $\beta$-relaxation peak was present, hence revealing the $\beta$-relaxation process. It is conceivable that the maximum contribution to $\epsilon^{\prime \prime}$ in the quenched sample, ${ }^{24,25}$ of magnitude $\sim 13 \times 10^{-5}$ at $\sim 205 \mathrm{~K}$, decreased, after annealing $o$-terphenyl for $24 \mathrm{~h}$ at $234 \mathrm{~K}$, enough to be overwhelmed by the contributions to $\epsilon^{\prime \prime}$ from it's $\alpha$-relaxation process, and that further annealing of the quenched glassy state of $o$-terphenyl would shift its $\alpha$-relaxation process to much lower frequencies, thereby decreasing the $\alpha$-relaxation process contributions at $T \sim 205 \mathrm{~K}$ enough to reveal a much smaller magnitude of the $\beta$-relaxation peak. The apparent vanishing of the $\beta$-relaxation peak in $o$-terphenyl may also be understood in the light of the Hikima et al. observations, ${ }^{20,21}$ that $o$-terphenyl nucleates rapidly and crystallizes during annealing at $T<T_{g}$ in the range where its $\beta$ relaxation occurs. Although this crystallization was not reported in their study, ${ }^{24,25}$ nucleation and crystallization can also decrease the already low $\epsilon^{\prime \prime}$ value of $13 \times 10^{-5}$ from all 
relaxation processes in $o$-terphenyl, and most sensitively decrease that due to the $\beta$-process.

\section{Combined time and temperature effects on localized motions}

The minimum observed in the plot of $\Delta \epsilon_{\beta}$ against $T$ in Fig. 3(B) needs to be understood in terms of the time-, and temperature-dependent changes in $\Delta \epsilon_{\beta}$. To do so, we may write the change in $\Delta \epsilon_{\beta}$ of a glass obtained by quenching a liquid as the sum of the changes due to the structural relaxation with time, and due to the increase in $T$ :

$$
d \Delta \epsilon_{\beta}=\left(\frac{\partial \Delta \epsilon_{\beta}}{\partial t}\right)_{T} d t+\left(\frac{\partial \Delta \epsilon_{\beta}}{\partial T}\right)_{t} d T,
$$

where the first term on the rhs of Eq. (3) represents the effect of structural relaxation of the glass at a fixed $T$, and the second term is due to the effects of the increase in $T$ at a formally fixed $t$. On dividing by $d T$,

$$
\frac{d \Delta \epsilon_{\beta}}{d T}=\left(\frac{\partial \Delta \epsilon_{\beta}}{\partial t}\right)_{T} \frac{1}{q}+\left(\frac{\partial \Delta \epsilon_{\beta}}{\partial T}\right)_{t},
$$

where $q(=d T / d t)$ is the heating rate used in obtaining the spectra, which was $0.1 \mathrm{~K} / \mathrm{min}(=1.67 \mathrm{mK} / \mathrm{s})$. The quantity $\left(\partial \Delta \epsilon_{\beta} / \partial t\right)_{T}$ has been found to be negative here [see Fig. $5(\mathrm{~B})]$, and elsewhere, $,, 6,22-27$ and the quantity $\left(\partial \Delta \epsilon_{\beta} / \partial T\right)_{t}$ to be positive. ${ }^{4-8,22-27}$ Thus, the minimum in the plot of Fig. 3(B) appears at a temperature when $q^{-1}\left(\partial \Delta \epsilon_{\beta} / \partial t\right)_{T}$ $=-\left(\partial \Delta \epsilon_{\beta} / \partial T\right)_{t}$ in Eq. (4). This means that the minimum would appear at a lower $T$ if $q$ is chosen to be low, and at a higher $T$ when $q$ is chosen to be high. In our study, this condition is satisfied at $145.6 \mathrm{~K}$. At $T<145.6 \mathrm{~K}$, structural relaxation dominates the change in $\Delta \epsilon_{\beta}$ with $T$, and at $T$ $>145.6 \mathrm{~K}$, the change in $\Delta \epsilon_{\beta}$ corresponds predominantly to its temperature dependence in the equilibrium state. Plots of the enthalpy of a glass obtained by rapid cooling are known to show a shallow minimum as a result of the time- and temperature-dependent structural relaxation, which is associated mainly with the $\alpha$-relaxation process. The $\Delta \epsilon_{\beta}$ against $T$ plot for the Johari-Goldstein relaxation for the glassy state obtained by rapid cooling appears in Fig. 3(B) to be qualitatively similar to such enthalpy plots. This seems remarkable as it shows that a relatively more rapid increase in the energy and entropy on heating above $T_{g}$ increases the rate of increase of $\Delta \epsilon_{\beta}$. In Fig. 4 of their study, Wagner and Richert ${ }^{38}$ have noted a more rapid increase in $\Delta \epsilon_{\beta}$ of salol beginning at a temperature about $30 \mathrm{~K}$ below its $T_{g}$. Olsen et al. . $^{22,23}$ have found a similar change in the slope but in an apparently continuous manner.

\section{Entropy and localized molecular motions}

Localized motions of the $\beta$-relaxation process also contribute to the anharmonic part of the entropy and to the configurational entropy of a glass, as do the faster modes of diffusion in the relaxation time distribution of the $\alpha$-relaxation process. ${ }^{11-13(\mathrm{c})}$ Since the relaxation rate of the $\beta$ process, $f_{m, \beta}$, has shown an Arrhenius-type temperature dependence, ${ }^{3-8}$ except for those in the recent studies on three glasses, where the slope of the plot increases on cooling at $T$ near $T_{g},{ }^{22,23}$ we may consider that for at least $T<T_{g}$, neither the decrease in the configurational entropy associated with the $\beta$-relaxation process, $S_{\text {conf, } \beta}$, nor that associated with the unfrozen modes of the $\alpha$-relaxation process, affects the $\beta$-relaxation kinetics. ${ }^{13(\mathrm{c})}$ In the entropy theory, a decrease in the net configurational entropy is seen to affect the $\alpha$-relaxation kinetics. ${ }^{39,40}$

We now consider how the proposed mechanism, ${ }^{34,35}$ namely, that all molecules contribute to the Johari-Goldstein relaxation, each reorienting by a temperature-independent small angle, affects the frozen-in configurational entropy of a glass. When all molecules (Avogadro number, $N_{\mathrm{A}}$, in one mole of a glass), contribute, $S_{\text {conf, } \beta}$ will be equal to $R \ln (n)$ where $R$ is the gas constant and $n$ the number of orientational sites available to one molecule, and would be less if less than $N_{\mathrm{A}}$ molecules contributed. In the simplest case, if there were only two orientational sites, then $S_{\text {conf, } \beta}$ would be equal to $5.76 \mathrm{~J} /(\mathrm{mol} \mathrm{K})$. Thus, the excess entropy of a glass over the crystal phase value, $S_{\text {exc }}$, minus the residual entropy, $S_{0}^{0}$, would be at least $5.76 \mathrm{~J} /(\mathrm{mol} \mathrm{K})$. The $S_{\text {exc }}$ data in Table I in Ref. 13(c) show that $\left(S_{\text {exc }}-S_{0}^{0}\right)$ of toluene, which has also been studied by ${ }^{2} \mathrm{H}$ NMR and dielectric measurements, is only $4.3 \mathrm{~J} /(\mathrm{mol} \mathrm{K})$ at $T$ near its $T_{g}$ of $\sim 117 \mathrm{~K}$, and it is lower at a lower $T$. Also, for several other liquids $\left(S_{\text {exc }}-S_{0}^{0}\right)$ is less than $5.76 \mathrm{~J} /(\mathrm{mol} \mathrm{K})$, as given in the respective Table $\mathrm{I}$ in Refs. 13(c) and Ref. 40. Clearly, the simple two-site model is inadequate for interpreting the entropy data of these glasses. This agrees with the Vogel and Rössler conclusion, which was reached by comparing the time scales of molecular jumps and the respective correlation time. ${ }^{34,35}$

\section{E. The time- and temperature-dependent relaxation rate}

The values of the distribution parameter, $\alpha$, plotted in Fig. 3(A) indicate a broad distribution of relaxation times. Because of the broadness of the spectra of the $\beta$-relaxation process at low temperatures, it has been difficult to determine the change in the relaxation rate, $f_{m, \beta}$, on annealing of the glass. Despite that, it is possible to deduce this information from the plots of $\ln \left(f_{m, \beta}\right)$ against $T$ or against $1 / T$. The change in $f_{m, \beta}$ with $T$ may be written as a sum of timedependent and temperature-dependent changes as follows, in a similar manner to $\Delta \epsilon_{\beta}$ :

$$
\frac{d \ln \left(f_{m, \beta}\right)}{d T}=\left(\frac{\partial \ln \left(f_{m, \beta}\right)}{\partial t}\right)_{T} \frac{1}{q}+\left(\frac{\partial \ln \left(f_{m, \beta}\right)}{\partial T}\right)_{t},
$$

and the change with $1 / T$ is given by

$$
\frac{d \ln \left(f_{m, \beta}\right)}{d(1 / T)}=\left(\frac{\partial \ln \left(f_{m, \beta}\right)}{\partial t}\right)_{T} \frac{d t}{d(1 / T)}+\left(\frac{\partial \ln \left(f_{m, \beta}\right)}{\partial(1 / T)}\right)_{t} .
$$

The first term on the rhs in Eqs. (5) and (6) represents the effect of structural relaxation and the second term the effect of thermal energy. Thus, during measurements on a quenched sample with increasing $T$, the slope of the plot of $\ln \left(f_{m, \beta}\right)$ against inverse temperature will be affected by the structural relaxation until a temperature is reached where the equilibrium state has been attained during the time of heating. Consequently, a change in the slope of the plot from the 
Arrhenius equation $\left[f_{m, \beta}=A \exp \left(-E^{*} / R T\right)\right.$, where $A$ is a constant, $E^{*}$ the Arrhenius energy, and $R$ is the gas constant], would be a reflection of a time-dependent effect on $f_{m, \beta}$ during the course of dielectric measurements, when the first term on the rhs in Eq. (6) is significant. The magnitude of $(1 / q)$ in the first term on the rhs of Eq. (5) is fixed at 600 $\mathrm{s} \mathrm{K}^{-1}$, and $\left[d t / d(1 / T)=\left(-T^{2} / q\right)\right]$ in the first term on the rhs of Eq. (6) decreases from $-7.88 \times 10^{6} \mathrm{~s} \mathrm{~K}$ at $114.6 \mathrm{~K}$, to $-15.2 \times 10^{6} \mathrm{~s} \mathrm{~K}$ at $159.1 \mathrm{~K}$. Therefore, a very small rate of increase in $f_{m, \beta}$ on spontaneous relaxation at a fixed $T$ would be substantially increased in magnitude on multiplication by the large values of $600 \mathrm{~s} / \mathrm{K}$ in Eq. (5) and -7.88 $\times 10^{6} \mathrm{~s} \mathrm{~K}^{-1}$ in Eq. (6). [It may be noted that $f_{m, \beta}$ obtained from the fixed frequency measurements differs from that obtained from the fixed temperature spectra, and therefore when $f_{m, \beta}$ obtained from such two sets is used to construct the plots of $\log \left(f_{m, \beta}\right)$ against $1 / T$, the slope of the plot changes as a result of inconsistency between the two sets of data analysis. This situation has been avoided here.]

An examination of the change in the slope of the plot in Fig. 4 shows that $f_{m, \beta}$ of the quenched state is lower than that expected for $f_{m, \beta}$ of the equilibrium state after structural relaxation has occurred, although the latter is denser. This indicates that the term $\left[\partial \ln \left(f_{m, \beta}\right) / \partial t\right]_{T}$ is positive. Since $[d t / d(1 / T)]$ is negative, the product of the two terms is negative, which adds to the negative second term in Eq. (6). The net result is that the slope of the Arrhenius plot is more negative when structural relaxation occurs during the measurements. To estimate its value at $131.6 \mathrm{~K}$ from the plot in Fig. 4, we extend the Arrhenius fit to the data from high temperatures to $131.6 \mathrm{~K}$, as shown by the dashed line. Knowing $[d t / d(1 / T)]$ from the heating rate, $\left[\partial \ln \left(f_{m, \beta}\right) / \partial(1 / T)\right]_{t}$ from the slope of the extended line, and $\left[d \ln \left(f_{m, \beta}\right) / d(1 / T)\right]$ from an estimate of the actual slope in Fig. 4 at $131.6 \mathrm{~K},\left[\partial \ln \left(f_{m, \beta}\right) / \partial t\right]_{T}$ at $131.6 \mathrm{~K}$ can be calculated. Its value is $\sim 4.924 \times 10^{-5} \ln (\mathrm{Hz}) \mathrm{s}^{-1}$.

A comparison of the actual $f_{m, \beta}$ values at $131.6 \mathrm{~K}$ shows that $f_{m, \beta}$ should increase on aging from $\sim 3.81 \mathrm{kHz}$ (from extrapolation of smoothed data used to find the slope at $131.6 \mathrm{~K})$ at the time the spectra was measured to an expected $4.56 \mathrm{kHz}$ at a formally infinite time, when spectra could be measured in the equilibrium state. This means that $\ln \left(f_{m, \beta}\right)$ must increase by $\sim 0.1797$, which should take roughly 3.6 ks. This is far shorter than the time scale of the isothermal anneal at $131.6 \mathrm{~K}$, which lasted $14.6 \mathrm{ks}$, yet during the anneal $f_{m, \beta}$ remained almost constant at $3.9 \mathrm{kHz}$. However, the stabilization time before the commencement of measurements was $\sim 1.8 \mathrm{ks}$ so $f_{m, \beta}$ should have achieved a constant value after about $1.8 \mathrm{ks}$ of annealing. Reexamining the $f_{m, \beta}$ data in light of this, the $f_{m, \beta}$ values obtained from spectra recorded before about $2 \mathrm{ks}$ seem to be slightly below average, but the data is very scattered. According to the annealing experiment at $131.6 \mathrm{~K}$, the equilibrium value of $f_{m, \beta}$ should be, at most, $4.2 \mathrm{kHz}$. The higher value obtained from extrapolation of the equilibrium line must be unreliable. The fact that $\Delta \epsilon_{\beta}$ attains an almost constant value during the isothermal anneal [see Fig. 5(B)] is further evidence that the sample reached equilibrium.

In summary, there are several possible reasons why an expected rise in $f_{m, \beta}$ was not observed during the annealing. First, in the glassy state of 5-methyl-2-hexanol at $131.6 \mathrm{~K}$, the equilibrium was reached quickly and therefore its consequences on $f_{m, \beta}$ were unobservable. In addition, the broadness of the $\epsilon^{\prime \prime}$ peak at low temperatures contributes significantly to the observed fluctuations in the fitting parameters, which obscure small time dependencies in $f_{m, \beta}$. Unfortunately, at $T<120 \mathrm{~K}$ where $f_{m, \beta}$ deviates increasingly more from the Arrhenius temperature dependence in Fig. 4, the time dependence of the spectra at a fixed temperature was not investigated.

Finally, it is likely that the increase in $f_{m, \beta}$ with time in this study was of the order of the changes in $f_{m, \beta}$ caused by a temperature instability of $0.1 \mathrm{~K}$ at $T=131.6 \mathrm{~K}$, and again this would tend to obscure any changes in $f_{m, \beta}$ with time. For that reason, we deduce that the slight curvature noted at lower temperatures for the data obtained during the heating of the quenched glass is a reflection of the time-dependent changes. Alternatively, the increase in the slope of the Arrhenius plot in Fig. 4 on heating at $T>140 \mathrm{~K}$ is a reflection of the attainment of the equilibrium state. The first terms in Eqs. (5) and (6) contribute significantly at low temperatures, but at high temperatures in the equilibrium liquid state this contribution vanishes.

One may also consider the alternative that the JohariGoldstein relaxation in this case shows deviations from the Arrhenius behavior at very low relaxation rates, where the magnitude of $\epsilon^{\prime \prime}$ spectral features are not well resolved, because of the limited frequency range and accuracy of the measurements. Perhaps measurements on those hyperquenched glasses whose $\epsilon^{\prime \prime}$ for the Johari-Goldstein relaxation remains large at temperatures far below $T_{g}$ would be well worth an investigation.

\section{F. The origin of the $\beta$-relaxation process}

The premise of at least two theories is that $\beta$ relaxation is a precursor of the $\alpha$-relaxation process, ${ }^{10,11}$ i.e., viscous flow or the $\alpha$-relaxation process would not occur unless the local motions of the $\beta$-relaxation process have occurred. Therefore, the time dependence of $\Delta \epsilon_{\beta}$ is important for developing a theory of viscous flow. As has been mentioned in Sec. I, there is only one relaxation process in the lowviscosity liquid at $\mathrm{GHz}$ frequencies. ${ }^{3-8}$ As the liquid is cooled and its density and viscosity increase, this relaxation process shifts to lower frequencies. After a certain density and viscosity has been reached on supercooling, the $\alpha$-relaxation process emerges from the low-frequency side of the $\beta$-relaxation peak and rapidly gains strength at the expense of the original relaxation process observed at $\mathrm{GHz}$ frequencies, which now becomes the faster relaxation. Concurrently the rate of the $\alpha$-relaxation process decreases progressively more rapidly on further supercooling until the liquid vitrifies. Thus, initially at a low viscosity, there is only one relaxation process with a relatively narrow distribution of relaxation times. At a high viscosity, there are two relaxation processes, each with a broad distribution of relaxation times. $^{4}$

Although this postulate could not be verified by supercooling a liquid, it has been verified by a different set of 
experiments in which the state of a liquid changed irreversibly with time isothermally as a result of the growth of a macromolecule in which covalent bonds replaced van der Waals' interactions. In such studies, it has been found that there is only one relaxation peak in the $\mathrm{GHz}$ frequency range in the low-density, low-viscosity liquid. ${ }^{41,42}$ As the liquid's density and viscosity increased as a result of the growth of the macromolecule at a constant temperature, the $\alpha$-relaxation process evolved, its peak continuously shifted to lower frequencies, and its strength increased. Concurrently, the peak of the original relaxation persisted at $\mathrm{GHz}$ frequencies without a significant shift in its frequency, but its dielectric relaxation strength decreased isothermally. The decrease in $\Delta \epsilon_{\beta}$ observed on structural relaxation, as seen in Fig. 5(B), admits to an equation similar to that observed for the decrease in the strength of the faster relaxation during the macromolecule's growth. ${ }^{43,44}$

The mechanism for the $\beta$-relaxation process was originally considered to be due to localized motions in regions of loose packing of molecules in the disordered structure, where no two molecules are identically placed. These regions were referred to as "islands of mobility" in an otherwise immobile disordered structure. ${ }^{5-7}$ It was also inferred that these islands collapse on structural relaxation, and $\Delta \epsilon_{\beta}$ decreases. ${ }^{27}$ Since the $\beta$-relaxation rate varies with temperature according to the Arrhenius equation, configurational entropy arising from localized motions would seem to play a minimum role in the mechanism of these localized motions.

In an alternative mechanism, originally by Williams and Watts, ${ }^{45,46}$ all molecules in a liquid and a glass contribute to the $\beta$-relaxation process, but by a small-angle reorientational diffusion. After this small-angle diffusion has occurred, a large-angle diffusion occurs. The latter constitutes the $\alpha$-relaxation process. This has been supported by solvation dynamics ${ }^{47}$ and NMR studies. ${ }^{34,35}$ This means that the rotational translational diffusion of molecules in a viscous liquid occurs in two steps: one by small-angle rotation and the second by large-angle rotation. A number of observations obtained from a study of metallic glasses ${ }^{48}$ and the glassy state of liquid crystals, ${ }^{49,50}$ are inconsistent with this interpretation. Also, a decrease in $\Delta \epsilon_{\beta}$ observed on cooling a glass and on its annealing indicates that if all molecules were to reorient by a small angle, the angle of reorientation would decrease on cooling and on structural relaxation, but the NMR studies have shown that this angle does not change. ${ }^{34,35}$

Alternatively, one may consider that a fraction of the total population of molecules ceases to reorient by a small angle on cooling and annealing. But this would entail that there is no precursor motion for the $\alpha$-relaxation of some molecules and therefore there would be a lesser contribution to both $\Delta \epsilon_{\beta}$ and $\Delta \epsilon_{\alpha}$. Consequently, the net contribution to the permittivity of the liquid, $\Delta \epsilon$, would decrease. But as an increase in $\Delta \epsilon$ has been observed on cooling, as is expected, ${ }^{28-31}$ it does not seem likely that at a high temperature all molecules contribute to the $\beta$-, and $\alpha$-relaxation processes and at low temperature only some do. Evidently, this alternative is not supported by the experiments. Thus one is left with the possibility that either the angle of reorientation of all molecules decreases if each molecule were to contrib- ute to $\Delta \epsilon_{\beta}$, or else only some molecules contribute to the $\beta$-relaxation process. The former is apparently not supported by the NMR studies. ${ }^{34,35}$

To summarize, there can be three occurrences that decrease $\Delta \epsilon_{\beta}$ : (i) all molecules contribute to the $\beta$-relaxation process, but the already small angle of reorientation of each molecule decreases further on cooling; (ii) the angle of reorientation does not decrease on cooling, but only some molecules contribute and their number decreases further on cooling; and (iii) both the population of such molecules and their reorientational angle decrease on cooling. Occurrence (i) is eliminated by the observations that the angle of reorientation is temperature independent, leaving us with (ii) and (iii) as plausible mechanisms.

A justification for the picture of the $\beta$-relaxation process as originating in the nonuniform structure of a glass containing randomly distributed regions of low density has been provided in Sec. 2.6 of Ref. 51. Briefly, one molecule or several molecules in such regions occupy sites with nonequivalent probabilities of orientations and/or positions, and molecules surrounding these regions are relatively fixed. Thus, there is a broad distribution of electrostatic field and field directions imposed by the relatively rigid surroundings of these molecules. This distribution perturbs the potential energy function and produces the features characteristic of the $\beta$-relaxation process.

Finally, it is worth deducing the implication of an equilibrium liquid's new entropy extrapolation to zero at $0 \mathrm{~K}^{52}$ for the $\beta$-relaxation process. In this extrapolation, the entropy of an equilibrium liquid decreases continuously from its value at $T>T_{g}$ to zero at $0 \mathrm{~K}$, and interpolation of the heat capacity has been used for this purpose. Accordingly, the number of minima in Goldstein's potential energy landscape ${ }^{53}$ configurational space or the number of inherent structures in the Stillinger-Weber energy landscape ${ }^{54}$ at a fixed pressure decreases as $T \rightarrow 0 \mathrm{~K}$, and only one minima becomes available, not accessible to the structure at $T$ $\rightarrow 0 \mathrm{~K}$, much like the arrangement in Penrose tiles, which has only one configuration. In this conjecture, the $\beta$-relaxation landscape should also vanish at $0 \mathrm{~K}$. Also, if $\beta$ relaxation was to be a precursor of the $\alpha$ relaxation, as is taken to be the premise in at least two theories, ${ }^{9,10}$ then the part of the landscape that expresses the $\beta$-relaxation process would vanish concurrently with the vanishing of the deep minimum, thereby meaning that all loosely packed regions in an equilibrium liquid gradually vanish on cooling an equilibrium liquid to $0 \mathrm{~K}$. This would not occur if all molecules contributed to the $\beta$-relaxation process at all temperatures by a temperature-independent small angle.

\section{CONCLUSIONS}

Structural relaxation of a glass decreases the orientational polarization due to the $\beta$-relaxation process, as does cooling. The relaxation rate of the $\beta$-relaxation process is also affected by structural relaxation, but much less, and is not directly observed here. The combined effects of the time, and temperature dependence produce a minimum in the plot of the dielectric relaxation strength of the $\beta$ process in the temperature plane, which shows that the structural relaxation 
effect dominates initially on heating and the temperature effect dominates ultimately. Hence, a plot of this relaxation strength resembles the structural relaxation plots of volume and enthalpy of a glass, and shows a shallow minimum. It is pointed out that the $\beta$-relaxation process may exist in equilibrium liquid $o$-terphenyl at $T>T_{g}$. Arguments based upon the temperature dependence of the magnitude of the orientational polarization show that a mechanism for $\beta$ relaxation in which each molecule performs a temperature-independent small-angle reorientation prior to a large-angle reorientation is inconsistent with the experimental observations.

The consequences of a description in which only some molecules in loosely packed regions, and not all molecules, contribute to the $\beta$-relaxation process, is consistent with dielectric results. It is expected that further local regions of loose packing may be created in the structure of a glass by high-energy irradiation, in a manner similar to the vacancies created in crystals. Similarity observed between the neutron and electron irradiation-caused defects in crystals and the $\beta$-relaxation thermodynamics has been discussed earlier. ${ }^{14}$ We suggest that the merit of the $\beta$-relaxation mechanism may be further determined by examining whether or not high-energy irradiation of a glass enhances the magnitude of its orientation polarization.

It is also possible to neutron-, or electron-irradiate those glasses that are found to crystallize at $T<T_{g}$ as a result of localized motions, ${ }^{20,21}$ and then determine whether the population of nucleation sites has grown as a result of irradiation. If their number has grown, the origin of crystallization is likely to be in the loosely packed or low-density regions in the disordered structure of a glass.

\section{ACKNOWLEDGMENTS}

One of the authors (G.P.J.) wishes to thank Trinity College Dublin, Ireland, for its hospitality during the period of this study. This research was supported by the International Collaborative Program of Enterprise Ireland, and a Natural Sciences and Engineering Council of Canada's grant to G.P.J. for general research. The studentship of G. P. was funded by INTEL, Ireland for work on low- $k$ dielectrics.

${ }^{1}$ I. M. Hodge, J. Non-Cryst. Solids 169, 211 (1994); Science 267, 1945 (1995).

${ }^{2}$ G. W. Scherer, J. Am. Ceram. Soc. 67, 504 (1984); Relaxation in Glass and Composites (Wiley, New York, 1986).

${ }^{3}$ G. P. Johari, J. Chim. Phys. Phys.-Chim. Biol. 82, 283 (1985).

${ }^{4}$ A. Kudlik, S. Benkhof, T. Blochowicz, C. Tschirwitz, and E. Rössler, J. Mol. Struct. 479, 201 (1999).

${ }^{5}$ G. P. Johari, J. Chem. Phys. 58, 1766 (1973); Ann. N.Y. Acad. Sci. 279, 117 (1976).

${ }^{6}$ G. P. Johari and M. Goldstein, J. Chem. Phys. 53, 2372 (1970).

${ }^{7}$ S. Kahle, J. Korus, E. Hempel, R. Unger, S. Höring, K. Schröter, and E. Donth, Macromolecules 30, 7214 (1997).
${ }^{8}$ C. Hansen, F. Stickel, T. Berger, R. Richert, and E. W. Fischer, J. Chem. Phys. 107, 1086 (1997).

${ }^{9}$ J. Y. Cavaille, J. Perez, and G. P. Johari, Phys. Rev. B 39, 2411 (1989).

${ }^{10}$ K. L. Ngai, Phys. Rev. E 57, 7346 (1998).

${ }^{11}$ M. Goldstein, J. Chem. Phys. 64, 4767 (1976); Ann. N.Y. Acad. Sci. 279, 68 (1976).

${ }^{12}$ P. D. Gujrati and M. Goldstein, J. Phys. Chem. 84, 859 (1980).

${ }^{13}$ (a) G. P. Johari, Ann. N.Y. Acad. Sci. 279, 102 (1976). (b) Philos. Mag. B 41, 41 (1981). (c) J. Chem. Phys. 112, 7518 (2000).

${ }^{14}$ G. P. Johari, Phys. Rev. B 33, 7201 (1986)

${ }^{15}$ R. C. Zeller and R. O. Pohl, Phys. Rev. B 4, 2029 (1971).

${ }^{16}$ H. Suga and S. Seki, Bull. Chem. Soc. Jpn. 46, 3020 (1973).

${ }^{17}$ H. Fujimori, M. Mizukami, and M. Oguni, J. Non-Cryst. Solids 204, 38 (1996).

${ }^{18}$ H. Fujimori and M. Oguni, J. Chem. Thermodyn. 26, 367 (1994).

${ }^{19}$ K. Hofer and G. P. Johari, J. Chem. Phys. 95, 2020 (1991).

${ }^{20}$ T. Hikima, M. Hanaya, and M. Oguni, Bull. Chem. Soc. Jpn. 69, 1863 (1996).

${ }^{21}$ T. Hikima, M. Hanaya, and M. Oguni, J. Mol. Struct. 479, 245 (1999).

${ }^{22}$ N. B. Olsen, J. Non-Cryst. Solids 235-237, 399 (1998).

${ }^{23}$ N. B. Olsen, T. Christensen, and J. C. Dyre, Phys. Rev. E 62, 4435 (2000).

${ }^{24}$ C. Hansen and R. Richert, Acta Polym. 48, 484 (1997).

${ }^{25} \mathrm{H}$. Wagner and R. Richert, J. Phys. Chem. B 103, 4071 (1999).

${ }^{26}$ J. Haddad and M. Goldstein, J. Non-Cryst. Solids 30, 1 (1978).

${ }^{27}$ G. P. Johari, J. Chem. Phys. 77, 4619 (1982), and references therein.

${ }^{28}$ C. P. Smyth, Dielectric Behavior and Structure (McGraw-Hill, New York, 1955).

${ }^{29}$ N. E. Hill, W. E. Vaughan, A. H. Price, and M. Davies, Dielectric Properties and Molecular Behaviour (Van Nostrand Reinhold, London, 1969), Chaps. 1 and 5.

${ }^{30}$ B. K. P. Scaife, Principles of Dielectrics (Clarendon, Oxford, 1998).

${ }^{31}$ N. G. McCrum, B. E. Read, and G. Williams, Anelastic and Dielectric Effects in Polymeric Solids (Wiley, New York, 1967).

${ }^{32}$ O. E. Kalinovskaya and J. K. Vij, J. Chem. Phys. 112, 3262 (2000).

${ }^{33}$ S. Havriliak Jr. and S. Negami, J. Polym. Sci., Part C: Polym. Symp. 14, 99 (1966).

${ }^{34}$ M. Vogel and E. Rössler, J. Phys. Chem. B 104, 4285 (2000).

${ }^{35}$ M. Vogel and E. Rössler, J. Chem. Phys. 114, 5802 (2000).

${ }^{36}$ J. Perez, J. Y. Cavaille, and L. David, J. Mol. Struct. 479, 183 (1999).

${ }^{37}$ U. Schneider, R. Brand, P. Lunkenheimer, and A. Loidl, Phys. Rev. Lett. 84, 5560 (2000).

${ }^{38}$ H. Wagner and R. Richert, J. Chem. Phys. 110, 11660 (1999).

${ }^{39}$ G. Adam and J. H. Gibbs, J. Chem. Phys. 43, 139 (1965).

${ }^{40}$ G. P. Johari, J. Chem. Phys. 112, 8958 (2000).

${ }^{41}$ D. A. Wasylyshyn, G. P. Johari, G. Salvetti, and E. Tombari, J. Phys.: Condens. Matter 9, 10521 (1997)

${ }^{42}$ D. A. Wasylyshyn, G. P. Johari, E. Tombari, and G. Salvetti, Chem. Phys. 223, 313 (1997)

${ }^{43}$ M. G. Parthun and G. P. Johari, J. Chem. Phys. 103, 7611 (1995).

${ }^{44}$ D. A. Wasylyshyn and G. P. Johari, J. Chem. Phys. 104, 5683 (1996).

${ }^{45}$ G. Williams and D. C. Watts, Trans. Faraday Soc. 67, 1971 (1971).

${ }^{46}$ G. Williams, Adv. Polym. Sci. 33, 60 (1979).

${ }^{47}$ H. Wagner and R. Richert, J. Non-Cryst. Solids 242, 19 (1998).

${ }^{48}$ A. Eisenberg and S. Reich, in Amorphous Materials, edited by R. W. Douglas and B. Ellis (Wiley, Chichester, 1972).

${ }^{49}$ G. P. Johari, Philos. Mag. B 46, 549 (1982).

${ }^{50}$ G. P. Johari and J. W. Goodby, J. Chem. Phys. 77, 5165 (1982).

${ }^{51}$ G. P. Johari, in Plastic Deformation of Amorphous and Semicrystalline Materials, edited by C. G'Sell and J. Perez (Les Edition de Physique, Les Ullis, France, 1983), p. 109.

${ }^{52}$ G. P. Johari, J. Chem. Phys. 113, 751 (2000). Chem. Phys. 265, 217 (2001).

${ }^{53}$ M. Goldstein, J. Chem. Phys. 51, 3728 (1969).

${ }^{54}$ F. Stillinger and T. A. Weber, Phys. Rev. A 28, 2408 (1983). 